\title{
Experience of Patients Undergoing Mini-Arthroscopy Compared to MRI in the Earliest Phases of Arthritis*
}

\author{
Maria J. H. de Hair ${ }^{1 \#}$, Marleen G. H. van de Sande ${ }^{1}$, Mario Maas², Danielle M. Gerlag ${ }^{1}$, Paul P. Tak ${ }^{1}$ \\ ${ }^{1}$ Department of Clinical Immunology and Rheumatology, Academic Medical Center, University of Amsterdam, Amsterdam, The \\ Netherlands; ${ }^{2}$ Department of Radiology, Academic Medical Center, University of Amsterdam, Amsterdam, The Netherlands. \\ Email: "m.j.dehair@amc.uva.nl \\ Received October $18^{\text {th }}, 2013$; revised November $10^{\text {th }}, 2013$; accepted December $1^{\text {st }}, 2013$ \\ Copyright (C) 2013 Maria J. H. de Hair et al. This is an open access article distributed under the Creative Commons Attribution Li- \\ cense, which permits unrestricted use, distribution, and reproduction in any medium, provided the original work is properly cited. In \\ accordance of the Creative Commons Attribution License all Copyrights @ 2013 are reserved for SCIRP and the owner of the intel- \\ lectual property Maria J. H. de Hair et al. All Copyright (C) 2013 are guarded by law and by SCIRP as a guardian.
}

\begin{abstract}
Objective: To evaluate the expectations and experience of patients undergoing mini-arthroscopy compared to contrast enhanced MRI for research purposes. Methods: Seventeen patients with early, active arthritis (Group A) and 21 autoantibody-positive individuals without any evidence of arthritis upon physical examination (Group B) were included. All subjects underwent both contrast enhanced MRI and synovial biopsy sampling by mini-arthroscopy of the same joint within one week. At inclusion and after both procedures, subjects filled in questionnaires with items about expectations and experience with regard to the procedures. Results: Before procedures, subjects in group B had a higher fear of and reluctance to undergo mini-arthroscopy compared to MRI ( $p<0.0001$ and $p=0.001$, respectively). Before procedures, $42 \%$ of the subjects preferred MRI, $11 \%$ of the subjects preferred mini-arthroscopy and $47 \%$ had no preference for either procedure. After both procedures, subjects preferences changed to 39\% for MRI, 32\% for mini-arthroscopy and $29 \%$ for no preference for one or the other procedure. When comparing Group A with Group B, there were no significant differences in preference before and after the procedures. Conclusion: Synovial biopsy sampling by mini-arthroscopy for analysis of synovial inflammation is a well-experienced procedure when compared to contrast enhanced MRI. These results support the use of mini-arthroscopy in a research setting from a patient perspective.
\end{abstract}

Keywords: Arthritis; Rheumatoid; Synovium; Arthroscopy; MRI

\section{Introduction}

Rheumatoid arthritis (RA) is a chronic autoimmune disease characterised by inflammation of synovial tissue leading to joint destruction and deformity [1]. Since the synovium is the main target tissue affected in RA, analysis of the features of the synovial inflammation is of major importance for pathogenetic studies. For analysis of synovial inflammation imaging but also histologic studies can be used. Analysis of the synovial tissue can be used to give insight into disease pathogenesis and to evaluate the effects of new treatments and the mechanisms of action of therapeutic compounds. Mini-arthroscopy, performed under local anaesthetics at the outpa-

\footnotetext{
*This study was financially supported by the Dutch Arthritis Association (grant 06-1-303) and the European Community's FP6 funding (Autocure)

\#Corresponding author.
}

tient clinic, is a feasible means of synovial biopsy sampling. It has been used for research purposes and is generally well tolerated with low complication rates [2-4]. Still, mini-arthroscopy is regarded as a rather invasive procedure and only performed in a few specialised centres.

Imaging of synovial inflammation can be done making use of MRI. MRI gives information about the degree of synovial inflammation, and additionally the compartment surrounding the synovium can be evaluated, including the bone (marrow) and cartilage. In the current project we performed dynamic contrast enhanced (DCE) MRI, by injecting a contrast agent intravenously during and after which time-dependent changes in MRI signal can be registered. DCE-MRI clearly visualizes the degree of synovial inflammation [5], enables to study physiologic characteristics of the inflamed synovium, such as vessel 
permeability, and has been shown to be a sensitive tool to detect changes after treatment [6-11].

At international scientific meetings, when results from studies using synovial biopsy sampling by mini-arthroscopy are presented by researchers of our Department of Clinical Immunology and Rheumatology of the Academic Medical Center (AMC) Amsterdam, questions are raised concerning patient's experience of synovial biopsy sampling by mini-arthroscopy, especially in individuals without arthritis. It seems that there is a general idea that mini-arthroscopy is an invasive procedure and a burden for patients, which seems to hamper the use of mini-arthroscopic synovial biopsy sampling in some research centres. Patient expectations and experience of mini-arthroscopy have never been studied. Therefore, we investigated patient's expectations before and experience after mini-arthroscopic synovial biopsy sampling and compared those with expectations before and experience after undergoing dynamic contrast-enhanced MRI, which is generally seen as a non-invasive procedure.

\section{Methods}

\subsection{Study Subjects}

Group A consisted of early arthritis patients (arthritis duration less than 1 year) with an inflamed knee, ankle or wrist, who were disease modifying antirheumatic drug naive (AMC's “Synoviomics” program) [12]. Group B consisted of individuals at risk for developing RA, defined by the presence of IgM-rheumatoid factor and/or anti-citrullinated protein antibodies, but no evidence of arthritis upon physical examination [13] (AMC's "PreSynoviomics” program) [14]. The study was performed according to the principles of the Declaration of Helsinki, approved by the medical ethical committee of the AMC, and all study subjects gave written informed consent.

\subsection{MRI}

All study subjects underwent DCE-MRI as previously described [6]. In Group A, a clinically inflamed (swollen and painful) wrist, knee or ankle joint was examined and in Group B an arbitrarily chosen knee joint was examined in all cases. Briefly, images were acquired on either a closed (1.5 Tesla GE Signa Horizon Echospeed, LX9.0, General Electric Medical Systems, Milwaukee, Wisconsin, USA) or open (Panorama 1 Tesla Open, Philips, Best, the Netherlands) MRI scanner, depending on the availability of the machine. Three scans were performed after which a contrast agent gadolinium (Magnevist, Schering, Berlin, Germany) was injected intravenously and 2 additional scans were performed. Total duration of the procedure was 60 minutes.

\subsection{Synovial Biopsy Sampling by Mini-Arthroscopy}

Within one week after the MRI, synovial biopsy sampling was performed at the outpatient clinic by means of mini-arthroscopy under local anaesthetics, as previously described [2,15]. The same joint was chosen for both procedures. For each study group 24 up to 32 synovial tissue biopsies were obtained during one procedure. The duration of the total procedure was 45 to 60 minutes.

\subsection{Questionnaires}

Before and after both procedures, subjects filled in questionnaires with items about expectations and the experience they had with regard to the procedures. Questions asked were 1) Do you have preference for MRI or miniarthroscopy or do you have "no preference"? 2) Please mark how well you think you are prepared for (a) MRI and (b) mini-arthroscopy 3) Please mark the level of fear you experience of (a) MRI and (b) mini-arthroscopy? 4) Please mark if you are reluctant to undergo (a) MRI and (b) mini-arthroscopy. The first question was multiple choice; the latter three questions were depicted on a visual analogue scale (VAS) of $0-100 \mathrm{~mm}$. In addition, study subjects could comment their choice of preference for one of the procedures. The first questionnaire was completed and handed in before and the second questionnaire was filled in after both procedures.

\subsection{Statistical Analysis}

We describe preference for either of the procedures before and after the procedures or compared preference in Group A with Group B using Chi-square test. In addition, differences in baseline emotional aspects with respect to both procedures and differences in preference after procedures compared to baseline were analysed using Wilcoxon signed rank test for related samples. P-value < 0.05 was considered statistically significant. Statistical analysis was performed using PASW Statistics 18 (SPSS Inc., Chicago, IL).

\section{Results}

Of 38 subjects baseline and follow-up questionnaires were available: 17 from Group A and 21 from Group B. Table 1 shows the disposition of study subjects with regard to type of joint examined and MRI machine used.

\subsection{Emotional Aspects}

With respect to emotional aspects subjects generally felt well prepared for both procedures. In Group B scores for fear and reluctance were higher for mini-arthroscopy compared to MRI, see Table 2. This was not the case for 
Table 1. Disposition of study subjects.

\begin{tabular}{ccc}
\hline Group A* $^{*}$ & Open MRI & Closed MRI \\
\hline Wrist & 0 & 1 \\
Knee & 2 & 10 \\
Ankle & 1 & 3 \\
\hline Group B & Open MRI & Closed MRI \\
\hline Knee & 6 & 15 \\
\hline
\end{tabular}

*Group A represents early arthritis patients; ${ }^{*}$ Group B represents autoantibody-positive individuals without arthritis at risk for developing RA.

Table 2. Emotional aspects regarding DCE-MRI and miniarthroscopy at baseline.

\begin{tabular}{cccc}
\hline Group A* $^{*}$ & Mini-arthroscopy & DCE-MRI & P-value \\
\hline $\begin{array}{c}\text { Preparation for } \\
\text { procedure }\end{array}$ & $85(52-91)$ & $82(60-94)$ & 0.504 \\
$\begin{array}{c}\text { Fear of procedure } \\
\text { Being reluctant to } \\
\text { undergo procedure }\end{array}$ & $25(6-46)$ & $5(0-51)$ & 0.084 \\
\hline Group B & $18(6-31)$ & $4(0-43)$ & 0.248 \\
\hline $\begin{array}{c}\text { Preparation for } \\
\text { procedure }\end{array}$ & $90(59-96)$ & $89(62-96)$ & 0.316 \\
$\begin{array}{c}\text { Fear of procedure } \\
\text { Being reluctant to } \\
\text { undergo procedure }\end{array}$ & $21(7-57)$ & $3(0-7)$ & $\mathbf{0 . 0 0 0}$ \\
\hline
\end{tabular}

DCE-MRI: dynamic contrast enhanced MRI; All items measured on a visual analogue scale of 0 - $100 \mathrm{~mm}$; Results depicted as median (IQR); "Group A represents early arthritis patients; " Group B represents autoantibody-positive individuals without arthritis at risk for developing RA.

Group A. However, there were no statistically significant differences between Groups A and B at baseline for individual emotional aspects (Table 3 ).

\subsection{Experience of Mini-Arthroscopy and DCE-MRI}

In the total study population, before undergoing both procedures $42 \%$ of the subjects preferred MRI, $11 \%$ preferred mini-arthroscopy and $47 \%$ had no preference for either procedure. After both procedures subjects preference changed to $39 \%$ preferring MRI, 32\% mini-arthroscopy and $29 \%$ having no preference for one of the procedures for studying synovitis. This shows that there was not a clear preference for one of the procedures. In addition, preference after both procedures was not significantly different from baseline preference $(\mathrm{P}=0.602)$.

When focusing on the subgroups, within Group A preference was as follows: at baseline $47 \%$ of the subjects did not have preference for either procedure, 35\% preferred MRI and $18 \%$ preferred mini-arthroscopy. After both procedures $29 \%$ did not have preference, $24 \%$ preferred MRI and 47\% preferred mini-arthroscopy (no difference was observed between preference after both procedures and before, $\mathrm{p}=0.755$ ).
Table 3. A comparison of emotional aspects regarding DCEMRI and mini-arthroscopy at baseline between group $A$ and group $B$.

\begin{tabular}{cccc}
\hline & Group A $^{*}$ & Group B $^{*}$ & P-value \\
\hline $\begin{array}{c}\text { Preparation for MRI } \\
\text { Preparation for } \\
\text { mini-arthroscopy }\end{array}$ & $82(60-94)$ & $89(62-96)$ & 0.521 \\
$\quad$ Fear of MRI & $5(52-91)$ & $90(59-96)$ & 0.293 \\
$\begin{array}{c}\text { Fear of mini-arthroscopy } \\
\text { Being reluctant to } \\
\text { undergo MRI }\end{array}$ & $25(6-46)$ & $21(0-7)$ & 0.318 \\
$\begin{array}{c}\text { Being reluctant to undergo } \\
\text { mini-arthroscopy }\end{array}$ & $18(0-43)$ & $1(0-5)$ & 0.751 \\
\hline
\end{tabular}

DCE-MRI: dynamic contrast enhanced MRI; All items measured on a visual analogue scale of 0 - $100 \mathrm{~mm}$; Results depicted as median (IQR); ${ }^{*}$ Group A represents early arthritis patients; "Group B represents autoantibody-positive individuals without arthritis at risk for developing RA.

Within group B, at baseline, 38\% did not have preference for either procedure, 57\% preferred MRI and 5\% preferred mini-arthroscopy. After both procedures, these percentages were, $29 \%, 52 \%$ and $19 \%$, respectively (no difference was observed between preference after both procedures and before $(\mathrm{P}=0.715)$. In addition, comparing study groups, there was no difference in preference between Groups A and B at baseline $(\mathrm{P}=0.271)$ or after both procedures $(\mathrm{P}=0.115)$.

Of importance, after both procedures, 6 individuals of Group A who did not have preference $(n=2)$ or preferred MRI $(n=4)$ at baseline changed to preference for mini-arthroscopy. Four of the individuals of Group B changed towards preference for mini-arthroscopy, of which 3 individuals preferred MRI at baseline. In both groups, none of the subjects who preferred mini-arthroscopy at baseline changed to MRI and, of those, only 1 individual changed to "no preference". See Figure 1 for preference at baseline, after both procedures and change in preference.

Of subjects who underwent MRI in the open scanner, nobody changed to preference for MRI afterwards. Main remarks with regard to DCE-MRI were complaints about the noise coming from the MRI machine and being immobile for a long period of time, the latter in particular in patients with arthritis. Some patients indicated that miniarthroscopy was better tolerated than expected, but two subjects complained about having more joint complaints until a few days after mini-arthroscopy. All study subjects were contacted by telephone one week after the procedures or consulted their rheumatologist within 3 weeks time and otherwise the procedures were well tolerated; no complications were reported.

\section{Discussion}

In this small study, we show that synovial biopsy sam- 

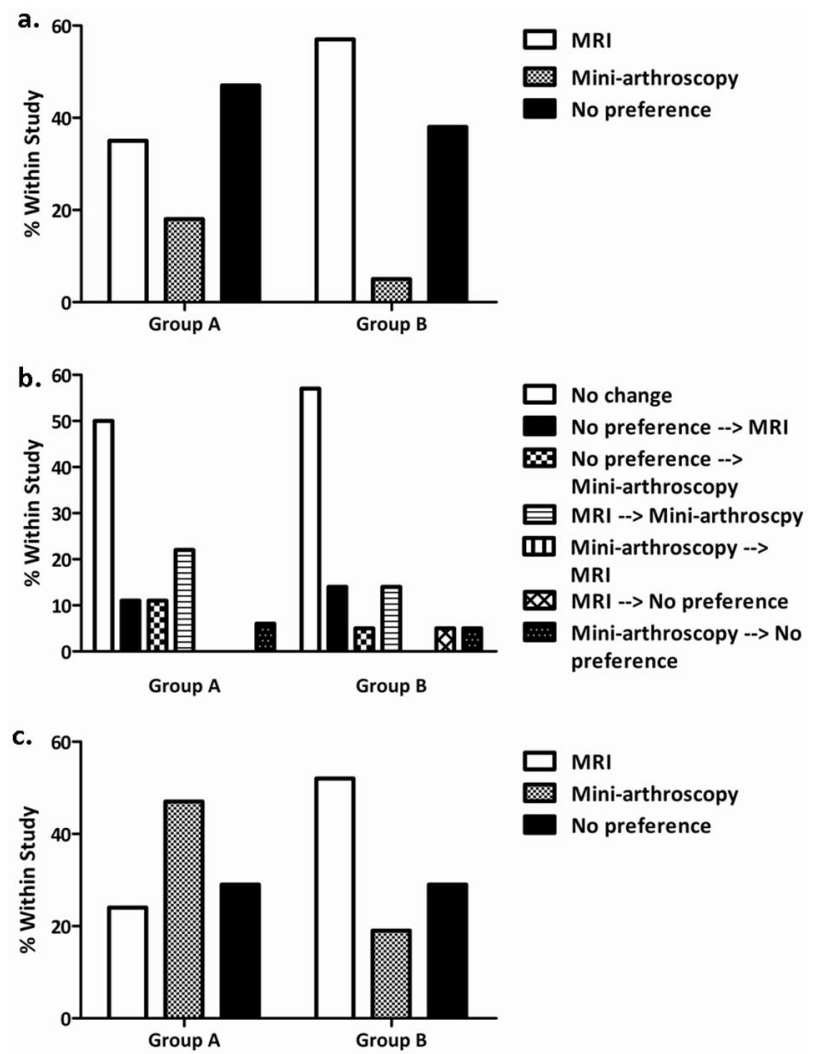

Figure 1. Preference of mini-arthroscopy or dynamic contrast enhanced MRI. Preference at baseline a, change in preference $b$ and preference after both procedures $c$. Results are depicted as percentage within study Group A or B. Group A represents early arthritis patients; Group B represents autoantibody-positive individuals without arthritis at risk for developing RA.

pling by means of mini-arthroscopy is well experienced when compared to DCE-MRI for studying synovial inflammation, both in early arthritis patients and in individuals without arthritis at risk for developing RA. Interestingly, we observed an increase, although not statistically significant, in the percentage of early arthritis patients preferring mini-arthroscopy after both procedures. Although at baseline the group of autoantibody-positive individuals without arthritis had higher levels of fear of and reluctance to undergo mini-arthroscopy than MRI, in this group the percentage of individuals preferring miniarthroscopy increased after procedures as well. Overall, these results refute assumptions that mini-arthroscopy would be a procedure not well-experienced by study subjects.

A factor that could be in favour of mini-arthroscopy may be that during mini-arthroscopy patients have direct contact with physicians and nurses, whereas during MRI they are completely on their own in a distinct room. After having undergone both procedures, most arthritis patients preferred mini-arthroscopy, which may be explained in part by the more stringent need for immobilisation during MRI. In contrast to the arthritis group, most individuals without arthritis still favoured MRI, which might be due to a short period of relative rest necessary after miniarthroscopy whereas after MRI no restrictions are imposed. Still, $19 \%$ of the subjects without arthritis favoured mini-arthroscopy after both procedures and none of these individuals preferring mini-arthroscopy at baseline changed to a preference for DCE-MRI. Of importance, the results of our study cannot be extrapolated to studies using conventional MRI, because scanning duration is generally longer for DCE-MRI and requires venipuncture in all cases, but do support the notion that mini-arthroscopy is generally well experienced, even in individuals without arthritis.

In summary, our results show the important observation that mini-arthroscopy, compared to DCE-MRI, is well experienced in patients with early arthritis as well as in autoantibody-positive individuals without arthritis who are at risk of developing RA. These results support the use of mini-arthroscopy in a research setting from a patient perspective, which, together with the low complication rates [2-4] should help to start using mini-arthroscopy in additional research centres.

\section{Acknowledgements}

We thank our study subjects for participation in the study and the AMC mini-arthroscopy team for synovial biopsy sampling. We thank the Dutch Arthritis Association (grant 06-1-303) and the European Community's FP6 funding (Autocure) for financial support.

\section{REFERENCES}

[1] P. P. Tak and B. Bresnihan, "The Pathogenesis and Prevention of Joint Damage in Rheumatoid Arthritis: Advances from Synovial Biopsy and Tissue Analysis," Arthritis \& Rheumatism, Vol. 43, No. 12, 2000, pp. 26192633.

http://dx.doi.org/10.1002/1529-0131(200012)43:12<2619 ::AID-ANR1>3.0.CO;2-V

[2] D. M. Gerlag and P. P. Tak, "How to Perform and Analyse Synovial Biopsies,” Best Practice \& Research Clinical Rheumatology, Vol. 23, No. 2, 2009, pp. 221232. http://dx.doi.org/10.1016/j.berh.2009.01.006

[3] D. Kane, D. J. Veale, O. Fitzgerald, et al., "Survey of Arthroscopy Performed by Rheumatologists," Rheumatology (Oxford), Vol. 41, No. 2, 2002, pp. 210-215. http://dx.doi.org/10.1093/rheumatology/41.2.210

[4] S. Vordenbaumen, L. A. Joosten, J. Friemann, et al., "Utility of Synovial Biopsy," Arthritis Research \& Therapy, Vol. 11, 2009, p. 256. http://dx.doi.org/10.1186/ar2847

[5] M. B. Axelsen, M. Stoltenberg, R. P. Poggenborg, et al., "Dynamic Gadolinium-Enhanced Magnetic Resonance 
Imaging Allows Accurate Assessment of the Synovial Inflammatory Activity in Rheumatoid Arthritis Knee Joints: A Comparison with Synovial Histology," Scandinavian Journal of Rheumatology, Vol. 41, No. 2, 2012, pp. 89-94. http://dx.doi.org/10.3109/03009742.2011.608375

[6] C. van der Leij, M. G. van de Sande and C. Lavini, et al., "Rheumatoid Synovial Inflammation: Pixel-by-Pixel Dynamic Contrast-Enhanced MR Imaging Time-Intensity Curve Shape Analysis-A Feasibility Study,” Radiology, Vol. 253, No. 1, 2009, pp. 234-240. http://dx.doi.org/10.1148/radiol.2531081722

[7] M. Navalho, C. Resende, A. M. Rodrigues, et al., "Dynamic Contrast-Enhanced 3-T Magnetic Resonance Imaging: A Method for Quantifying Disease Activity in Early Polyarthritis,” Skeletal Radiology, Vol. 41, No. 1, 2012, pp. 51-59.

http://dx.doi.org/10.1007/s00256-011-1112-8

[8] B. Ejbjerg, E. Narvestad, E. Rostrup, et al., "Magnetic Resonance Imaging of Wrist and Finger Joints in Healthy Subjects Occasionally Shows Changes Resembling Erosions and Synovitis as Seen in Rheumatoid Arthritis," Arthritis \& Rheumatism, Vol. 50, 2004, pp. 1097-1106. http://dx.doi.org/10.1002/art.20135

[9] M. Ostergaard, I. Lorenzen and O. Henriksen, "Dynamic Gadolinium-Enhanced MR Imaging in Active and Inactive Immunoinflammatory Gonarthritis,” Acta Radiologica, Vol. 35, No. 3, 1994, pp. 275-281.

[10] M. Ostergaard, M. Stoltenberg, P. Lovgreen-Nielsen, et al., "Quantification of Synovistis by MRI: Correlation between Dynamic and Static Gadolinium-Enhanced Magnetic Resonance Imaging and Microscopic and Macro- scopic Signs of Synovial Inflammation,” Magnetic Resonance Imaging, Vol. 16, No. 7, 1998, pp. 743-754. http://dx.doi.org/10.1016/S0730-725X(98)00008-3

[11] A. L. Tan, S. F. Tanner, P. G. Conaghan, et al., "Role of Metacarpophalangeal Joint Anatomic Factors in the Distribution of Synovitis and Bone Erosion in Early Rheumatoid Arthritis,” Arthritis \& Rheumatism, Vol. 48, No. 5, 2003, pp. 1214-1222. http://dx.doi.org/10.1002/art.10963

[12] M. J. de Hair, L. C. Harty, D. M. Gerlag, et al., "Synovial Tissue Analysis for the Discovery of Diagnostic and Prognostic Biomarkers in Patients with Early Arthritis," The Journal of Rheumatology, Vol. 38, No. 9, 2011, pp. 2068-2072. http://dx.doi.org/10.3899/jrheum.110426

[13] D. M. Gerlag, K. Raza, L. G. van Baarsen, et al., "EULAR Recommendations for Terminology and Research in Individuals at Risk of Rheumatoid Arthritis: Report from the Study Group for Risk Factors for Rheumatoid Arthritis,” Annals of the Rheumatic Diseases, Vol. 71, No. 5, 2012, pp. 638-641. http://dx.doi.org/10.1136/annrheumdis-2011-200990

[14] M. G. van de Sande, M. J. de Hair, C. van der Leij, et al., "Different Stages of Rheumatoid Arthritis: Features of the Synovium in the Preclinical Phase," Annals of the Rheumatic Diseases, Vol. 70, No. 5, 2011, pp. 772-777. http://dx.doi.org/10.1136/ard.2010.139527

[15] M. G. van de Sande, "Evaluating Antirheumatic Treatments Using Synovial Biopsy: A Recommendation for Standardisation to Be Used in Clinical Trials," Annals of the Rheumatic Diseases, Vol. 70, No. 3, 2011, pp. 423427. http://dx.doi.org/10.1136/ard.2010.139550 